

\title{
Numerical modeling of floating potentials in electrokinetic problems using an asymptotic method
}

Damien Voyer, Sergio Corridore, Annabelle Collin, Riccardo Scorretti, Clair Poignard

\section{- To cite this version:}

Damien Voyer, Sergio Corridore, Annabelle Collin, Riccardo Scorretti, Clair Poignard. Numerical modeling of floating potentials in electrokinetic problems using an asymptotic method. IEEE Transactions on Magnetics, 2020, 10.1109/TMAG.2019.2951942 . hal-02467851

\section{HAL Id: hal-02467851 \\ https://hal.science/hal-02467851}

Submitted on 7 Feb 2020

HAL is a multi-disciplinary open access archive for the deposit and dissemination of scientific research documents, whether they are published or not. The documents may come from teaching and research institutions in France or abroad, or from public or private research centers.
L'archive ouverte pluridisciplinaire HAL, est destinée au dépôt et à la diffusion de documents scientifiques de niveau recherche, publiés ou non, émanant des établissements d'enseignement et de recherche français ou étrangers, des laboratoires publics ou privés. 


\section{Numerical modeling of floating potentials in electrokinetic problems using an asymptotic method}

\begin{tabular}{|r|l|}
\hline Journal: & Transactions on Magnetics - Conferences \\
\hline Manuscript ID & MAGCON-19-08-0212.R1 \\
\hline Manuscript Type: & Compumag 2019 \\
\hline Author: & n/a \\
\hline Complete List of Authors: & $\begin{array}{l}\text { Voyer, Damien; EIGSI La Rochelle Engineering School } \\
\text { Corridore, Sergio; INRIA } \\
\text { Collin, Annabelle; INRIA } \\
\text { Scorretti, Riccardo; Laboratoire Ampère } \\
\text { Poignard, Clair; INRIA Bordeaux-Sud Ouest, Applied Mathematics }\end{array}$ \\
\hline Keywords: & 72 Numerical methods \\
\hline &
\end{tabular}

\section{SCHOLARONE ${ }^{\text {M }}$ \\ Manuscripts}




\title{
Numerical modeling of floating potentials in electrokinetic problems using an asymptotic method
}

\author{
D. Voyer ${ }^{1,2}$, S. Corridore ${ }^{2}$, A. Collin ${ }^{2}$, R. Scorretti ${ }^{3}$ and C. Poignard ${ }^{2}$ \\ ${ }^{1}$ EIGSI La Rochelle, La Rochelle Cedex F-17041, France, voyer@eigsi.fr \\ ${ }^{2}$ Team MONC, INRIA, CNRS UMR 5251, Bordeaux INP, Talence Cedex F-33405, France \\ ${ }^{3}$ Université de Lyon, Ampère, UMR 5005, Ecully Cedex F-69134, France
}

\begin{abstract}
Floating potentials appear in electrokinetic problems when isolated high conductive materials are included in a dielectric or weakly conductive ambient medium. The large contrast of conductivities generates numerical issues that make hard the computation of the electric potential. The paper proposes a rigorous numerical method to tackle such kind of problems. Interestingly, a correction to the case of perfect conductor is given in order to improve the accuracy of the computation. The method involves a cascade of two elementary problems set respectively in the ambient medium and in the high conductive inclusions. An example is proposed with a 4-electrode system designed to both induce electroporation in a biological tissue sample and measure the resulting impedance. The approach is extended to a nonlinear problem by taking advantage of the iterative scheme that is necessarily applied in this case.
\end{abstract}

Index Terms - Floating potential, asymptotic method, nonlinear problem, 4-electrode system.

\section{INTRODUCTION}

$\mathrm{T}$ HE concept of floating potential is encountered in electrokinetic problems when isolated conductors of high conductivity are embedded in a dielectric or low conductive medium [1]. One can mention for example earthing systems where metal rods are buried into the ground [2] or 4-electrode systems that are often used to measure the impedance of biological tissues [3]. The direct solution of this kind of problems has two main drawbacks: this approach requires the meshing of the interior of the conductors and the high contrast of conductivities leads to numerical issues in the matrix inversion [4]. Replacing the electrodes by perfect conductors provides a reasonable approximation. The resulting problem leads to an additional degree of freedom related to the isopotential value on the surface of the perfect conductor. However, the solution can be computed rigorously without modification of the matrix system using superposition of solutions $[5,6]$ or iterative techniques $[2,7]$.

In this paper, we show that a more accurate approximation can be achieved thanks to an asymptotic expansion of the potential. Asymptotic expansions are formal series with respect to a small parameter (here the ratio of conductivities), which provide an accurate and rigorous approximation of the solution (here the potential). In the case of floating potentials, the zero order solution (the first term of the expansion) is shown to coincide with the perfectly conductor case. The potential is then approached one step further with the first order correction (second term of the expansion). It implies two successive problems defined respectively in the ambient medium and in the high conductive inclusions, which avoids describing the high contrast of conductivities in a single problem.

The paper is organized as follows: the formal asymptotic expansion of the solution to the electrokinetic problem is given in Section II. In section III, the numerical convergence with respect the conductivity ratio is studied in a 2D-toy configuration. The method is then applied to a $3 \mathrm{D}$ example that shows the consistency of the method, while a direct computation leads to unsatisfactory results. Finally, a nonlinear case is proposed in section IV in the 2D configuration.

\section{FORMULATION OF THE ELECTROKINETIC PROBLEM}

We consider the model of a 4-electrode system dedicated to measure the impedance of a biological tissue. In this device, a current is injected through both external electrodes inside the biological tissue. In the same time, both internal electrodes are connected to a high impedance in the external circuit; consequently, no current is supposed to flow through them. Internal electrodes are said to be passive while external ones are said to be active. The impedance of the biological tissue is then calculated measuring the potential difference induced between the internal passive electrodes that do not suffer from to voltage drop due to the electrode/tissue interfacial impedance [3].

The general model of a symmetric 4-electrode system with two pairs of active and passive electrodes is shown in Figure 1.

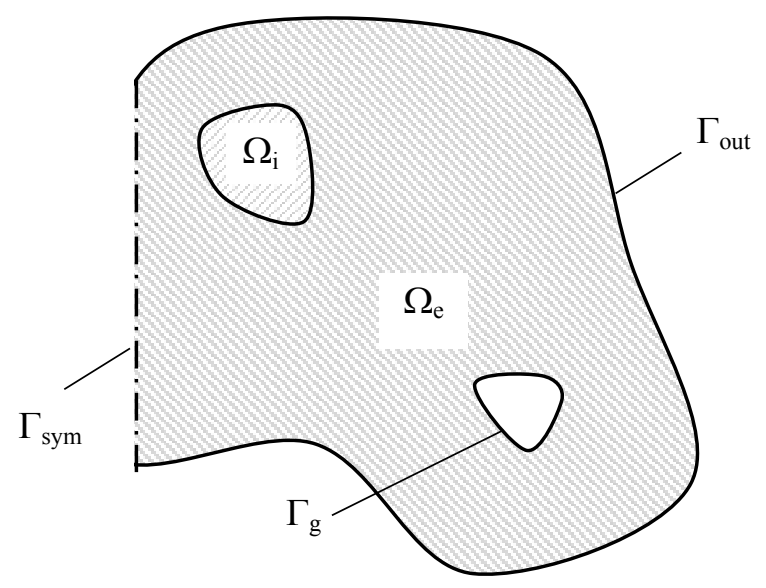

Figure 1: different domains considered in a symmetric 4-electrode device 
The initial problem to solve reads:

$$
\left\{\begin{array}{l}
-\nabla \cdot\left(\sigma_{e} \nabla \varphi_{e}\right)=0 \text { in } \Omega_{e} \\
\varphi_{e \mid \Gamma_{g}}=V \quad \varphi_{e \mid \Gamma_{\text {sym }}}=0 \quad \sigma_{e} \partial_{n} \varphi_{e \mid \Gamma_{\text {out }}}=0 \\
-\nabla \cdot\left(\sigma_{i} \nabla \varphi_{i}\right)=0 \text { in } \Omega_{i} \\
\varphi_{i \mid \partial \Omega_{i}}=\varphi_{e \mid \partial \Omega_{i}} \quad \sigma_{e} \partial_{n} \varphi_{e \mid \partial \Omega_{i}}=\sigma_{i} \partial_{n} \varphi_{i \mid \partial \Omega_{i}}
\end{array}\right.
$$

where $\Gamma_{\mathrm{g}}$ is the border for the active electrode, $\Gamma_{\text {out }}$ the external border of the biological tissue and $\Gamma_{\text {sym }}$ the symmetry plane. $\sigma_{\mathrm{e}}$ is the conductivity of the biological tissue defined inside the domain $\Omega_{\mathrm{e}}$ and $\sigma_{\mathrm{i}}$ the conductivity of the passive electrodes defined inside the domains $\Omega_{\mathrm{i}}$.

The solution for $\varphi_{\mathrm{e}}$ and $\varphi_{\mathrm{i}}$ can be expanded using formal series with respect to the parameter $\varepsilon=\sigma_{\mathrm{e}} / \sigma_{\mathrm{i}}<<1$ :

$$
\left\{\begin{array}{l}
\varphi_{e}=\varphi_{e}^{0}+\varepsilon \varphi_{e}^{1}+\cdots \\
\varphi_{i}=\alpha_{0}+\varepsilon\left(\varphi_{i}^{1}+\alpha_{1}\right)+\cdots
\end{array}\right.
$$

where the terms $\alpha_{\mathrm{k}}$ are constant and $\left\{\varphi_{\mathrm{i}}^{\mathrm{k}}, \varphi_{\mathrm{e}}^{\mathrm{k}}\right\}$ solve a differential problem set respectively in $\Omega_{\mathrm{i}}$ and $\Omega_{\mathrm{e}}$. Identifying the terms with the same power in $\varepsilon$, we infer the cascade of problems. The first problem to solve appears to be perfect conductor case. The couple $\left(\varphi_{\mathrm{e}}{ }^{0}, \alpha_{0}\right)$ is the solution to

$$
\left\{\begin{array}{c}
-\nabla \cdot\left(\sigma_{e} \nabla \varphi_{e}^{0}\right)=0 \text { in } \Omega_{e} \\
\varphi_{e \mid \Gamma_{g}}^{0}=V \quad \varphi_{e \mid \Gamma_{\text {sym }}}^{0}=0 \quad \sigma_{e} \partial_{n} \varphi_{e \mid \Gamma_{\text {out }}}^{0}=0 \\
\varphi_{e \mid \partial \Omega_{i}}^{0}=\alpha_{0} \text { with } \alpha_{0} \text { such that } \int_{\partial \Omega_{i}} \sigma_{e} \partial_{n} \varphi_{e}^{0} d s=0
\end{array}\right.
$$

The solution $\left(\varphi_{\mathrm{e}}^{0}, \alpha_{0}\right)$ is computed by superposing two solutions of problems where the homogeneous Dirichlet condition is set respectively on the boundary $\partial \Omega_{\mathrm{i}}$ of the passive electrode and on the boundary $\Gamma_{\mathrm{g}}$ of the active electrode. More precisely, consider the two following problems:

$$
\begin{aligned}
& \begin{cases}-\nabla .\left(\sigma_{e} \nabla \lambda\right)=0 \text { in } \Omega_{e} \\
\lambda_{\mid \Gamma_{g}}=V \quad \lambda \mid \partial \Omega_{i} \cup \Gamma_{\text {sym }}=0 & \sigma_{e} \partial_{n} \lambda_{\mid \Gamma_{\text {out }}}=0\end{cases} \\
& \left\{\begin{array}{ll}
-\nabla \cdot\left(\sigma_{e} \nabla \mu\right)=0 \text { in } \Omega_{e} \\
\mu_{\mid \partial \Omega_{i}}=1 \quad \mu_{\mid \Gamma_{g}} \cup \Gamma_{\text {sym }}=0
\end{array} \quad \sigma_{e} \partial_{n} \mu_{\mid \Gamma_{\text {out }}}=0\right.
\end{aligned}
$$

Then $\varphi_{\mathrm{e}}{ }^{0}=\lambda+\alpha_{0} \mu$ in $\Omega_{\mathrm{e}}$ and $\varphi_{\mathrm{i}}{ }^{0}=\alpha_{0}$ in $\Omega_{\mathrm{i}}$ with

$$
\alpha_{0}=-\int_{\partial \Omega_{i}} \sigma_{e} \partial_{n} \lambda d s / \int_{\partial \Omega_{i}} \sigma_{e} \partial_{n} \mu d s
$$

or equivalently

$$
\alpha_{0}=-\int_{\Omega_{e}} \sigma_{e} \nabla \lambda \nabla \mu d x / \int_{\Omega_{e}} \sigma_{e}|\nabla \mu|^{2} d x
$$

To improve the zero order approximation, the terms $\left\{\varphi_{\mathrm{i}}^{1}, \varphi_{\mathrm{e}}{ }^{1}, \alpha_{1}\right\}$ satisfy similar problems as (3) with $\left\{\varphi_{\mathrm{i}}^{0}, \varphi_{\mathrm{e}}{ }^{0}\right\}$ as source terms:

$$
\begin{aligned}
& \left\{\begin{array}{l}
-\nabla \cdot\left(\sigma_{i} \nabla \varphi_{i}^{1}\right)=0 \text { in } \Omega_{i} \\
\partial_{n} \varphi_{i \mid \partial \Omega_{i}}^{1}=\partial_{n} \varphi_{e \mid \partial \Omega_{i}}^{0} \quad \int_{\partial \Omega_{i}} \varphi_{i}^{1}=0
\end{array}\right. \\
& \left\{\begin{array}{l}
-\nabla \cdot\left(\sigma_{e} \nabla \varphi_{e}^{1}\right)=0 \text { in } \Omega_{e} \\
\varphi_{e \mid \Gamma_{g} \cup \Gamma_{s y m}}^{1}=0 \quad \sigma_{e} \partial_{n} \varphi_{e \mid \Gamma_{\text {out }}}^{1}=0 \\
\varphi_{e \mid \partial \Omega_{i}}^{1}=\varphi_{i \mid \partial \Omega_{i}}^{1}+\alpha_{1} \text { with } \alpha_{1} \text { such that } \int_{\partial \Omega_{i}} \sigma_{e} \partial_{n} \varphi_{e}^{1}=0
\end{array}\right.
\end{aligned}
$$

The numerical problem (7a) is solved using a Lagrange multiplier to ensure the Gauge condition. Instead of solving (7b), one solves

$$
\left\{\begin{array}{l}
-\nabla \cdot\left(\sigma_{e} \nabla \chi\right)=0 \text { in } \Omega_{e} \\
\chi\left|\partial \Omega_{i}=\varphi_{i \mid \partial \Omega_{i}}^{1} \quad \chi\right| \Gamma_{g} \cup \Gamma_{\text {sym }}=0 \quad \sigma_{e} \partial_{n} \chi \chi_{\Gamma_{\text {out }}}=0
\end{array}\right.
$$

The constant $\alpha_{1}$ is given by substituting $\lambda$ by $\chi$ in (6). The first order correction is then given by $\varphi_{\mathrm{e}}^{1}=\chi+\alpha_{1} \mu$ in $\Omega_{\mathrm{e}}$.

\section{III.NUMERICAL RESULTS}

\section{A. Linear case}

A 2D model of a symmetric 4-electrode system is first introduced in order to perform the convergence study. The problem is solved using the finite element method with a coarse, medium and fine meshes (see Fig. 2; note that the dimensions are realistic). The number of degree of freedoms (dof) for solving directly the initial problem (1) is 4300,67000 and 1060000 for the coarse, medium and fine meshes. The above potential $\left\{\varphi_{\mathrm{i}}^{\mathrm{k}}, \varphi_{\mathrm{e}}^{\mathrm{k}}\right\}, \mathrm{k}=0,1$ are easier to compute since there is no high contrast in the conductivities and only a single domain is involved in the different problems $\left(\Omega_{\mathrm{i}}\right.$ or $\left.\Omega_{\mathrm{e}}\right)$. In particular, the number of dof in the domain $\Omega_{\mathrm{e}}$ (resp. in the domain $\Omega_{\mathrm{i}}$ ) is 4100 (respectively 200), 65000 (respectively 2000) and 1020000 (respectively 40000 ) for the coarse, medium and fine meshes.

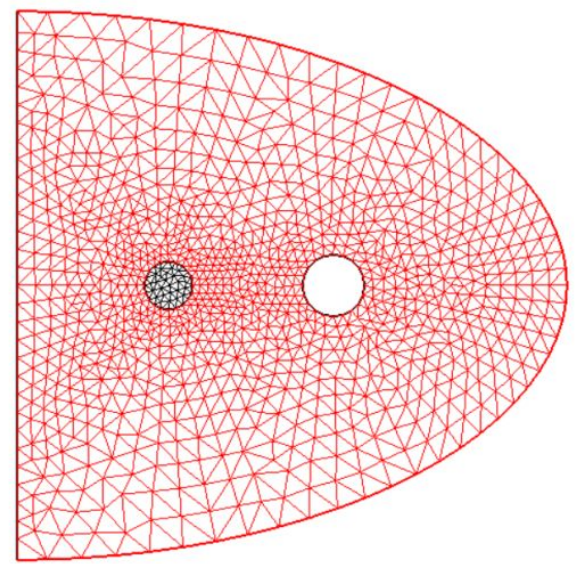

Fig.2: 2D model of the 4-electrode system with a coarse mesh. The diameter for the active (resp. passive) electrodes is $0.45 \mathrm{~mm}$ (resp. $0.35 \mathrm{~mm}$ ) and the separation distance between negative and positive electrodes is $4.6 \mathrm{~mm}$ (resp. $2.2 \mathrm{~mm})$.

A 4-electrode device is designed to measure the resistance $\mathrm{R}$ defined as the ratio of the potential difference $U$ between the passive electrode and the current $I$ flowing through the active 
electrodes. Since the passive electrodes are not perfect conductors, the mean potential at the surface of the passive electrodes is considered to compute the resistance:

$$
\begin{aligned}
R=\frac{U}{I} & =\frac{2 \int \Omega_{\partial \Omega_{i}} \varphi_{i} d s}{\left|\partial \Omega_{i}\right| \int_{\Gamma_{g}} \sigma_{e} \partial_{n} \varphi_{e}} \\
& =\frac{2 V \quad \int_{\partial \Omega_{i}} \varphi_{i} d s}{\left|\partial \Omega_{i}\right| \int_{\Omega_{e}} \sigma_{e}\left|\nabla \varphi_{e}\right|^{2} d x+\int_{\Omega_{i}} \sigma_{i}\left|\nabla \varphi_{i}\right|^{2} d x}
\end{aligned}
$$

where the factor 2 is introduced because of the symmetry in the initial problem.

Using the asymptotic expansion, $R$ is approached by

$$
\begin{aligned}
R= & \frac{2 V}{\int_{\Omega_{e}} \sigma_{e}\left|\nabla \varphi_{e}^{0}\right|^{2} d x}\left\{\alpha_{0}+\right. \\
& \left.\varepsilon\left(\alpha_{1}-\alpha_{0} \frac{2 \int_{\Omega_{e}} \nabla \varphi_{e}^{0} \nabla \varphi_{e}^{1}+\int_{\Omega_{i}}\left|\nabla \varphi_{i}^{1}\right|^{2}}{\int_{\Omega_{e}}\left|\nabla \varphi_{e}^{0}\right|^{2} d x}\right)\right\}+O\left(\varepsilon^{2}\right)
\end{aligned}
$$

In (9), the leading term of $\mathrm{R}$ depends only on the couple $\left(\varphi_{\mathrm{e}}^{0}, \alpha_{0}\right)$ while $\left(\varphi_{\mathrm{e}}^{0}, \alpha_{0}\right)$ and $\left(\varphi_{\mathrm{i}}^{1}, \alpha_{1}\right)$ are involved at the second order of accuracy. The next paragraph deals with this numerical convergence study.

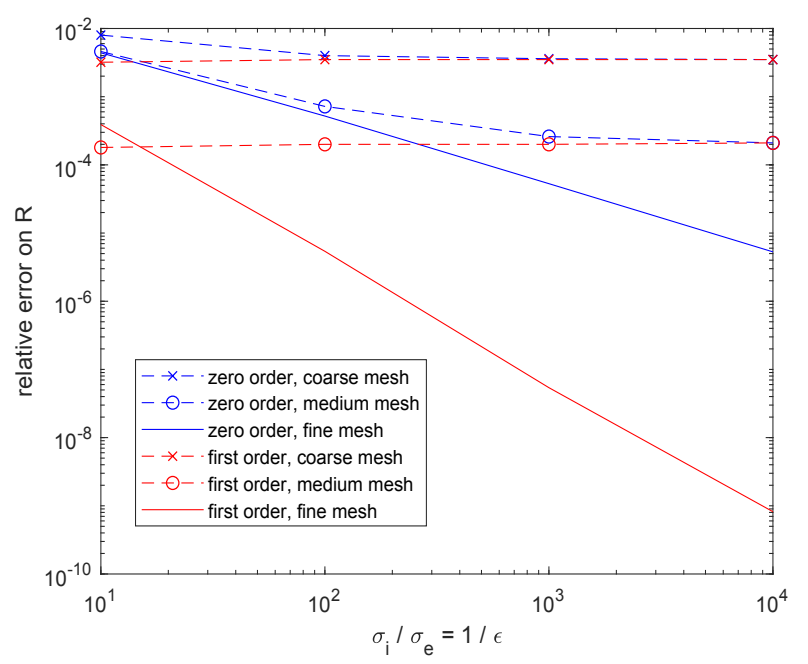

Fig.3: Convergence of the asymptotic approach at the zero and first orders for three different meshes with the $2 \mathrm{D}$ model

The resistance $\mathrm{R}$ is computed using (8) for a tissue with a constant conductivity $\sigma_{\text {e. }}$ Fig. 3 reports the relative error of the asymptotic development with respect to $\varepsilon$ at the zero and first orders. Since the exact solution is not analytical, the reference value is chosen as the numerical solution of the direct computation with the fine mesh. One observes that when the asymptotic method is performed with the fine mesh, the relative error on $\mathrm{R}$ decreases for the zero order computation following the rate $\varepsilon$. Adding the first order correction, the relative error decreases following a rate slightly lower than $\varepsilon^{2}$. Those observations are in agreement with the theoretical result given by (9). However, when the mesh is coarser, the accuracy of the finite element solution is not sufficient enough and the error due to the mesh is greater than the error due to the truncation of the asymptotic expansion. Thus, the relative error on $\mathrm{R}$ does not decrease under $3.510^{-3}$ (respectively $2.1 \quad 10^{-4}$ ) for the coarse (respectively medium) mesh in Fig. 3: this corresponds exactly to the relative error on $\mathrm{R}$ obtained with the direct computation performed on the same mesh.

A 3D model of the symmetric 4-electrode system has been simulated in order to get closer to the real device. The mesh shown Fig. 4 is such that there are 3200000 dof to solve the initial problem; the number of dof in the domain $\Omega_{\mathrm{e}}$ (respectively in the domain $\Omega_{\mathrm{i}}$ ) is 1200000 (2 100000 ). In these conditions, the computation time to solve both zero and first order problems with the asymptotic method is reduced by $20 \%$ compared to the time required to solve the direct problem. The simulation performed when $\varepsilon=10^{-1}$ gives a relative error on R of $5.010^{-3}$ for the zero order and $5.510^{-3}$ for the first order; the error decreases to $1.110^{-3}$ for the zero order and $5.610^{-4}$ for the first order when $\varepsilon=10^{-2}$. The comparison cannot be performed further because of the limitation of the numerical resources. Moreover, the matrix of the initial problem becomes numerically singular due to the high contrast of conductivities and the numerical inversion is not accurate.

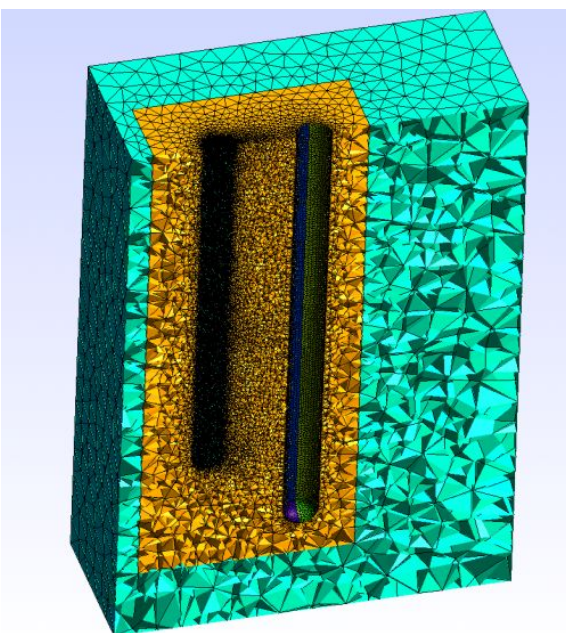

Fig.4: 3D model of the symmetric 4-electrode system with the same dimensions as in Fig. 2. The height of the active (respectively passive) electrodes is $6.3 \mathrm{~mm}$ (respectively $5.2 \mathrm{~mm}$ ).

\section{B. Nonlinear case}

When the 4-electrode system is used to monitor the tissue impedance during electroporation, the problem becomes nonlinear since the tissue conductivity depends on the electric field amplitude. In this section, a sigmoid function is introduced to model the dependence of the conductivity $\sigma_{\mathrm{e}}$ with respect to the electric field as it is generally considered in the electroporation phenomenon [8]:

$$
\sigma_{e}\left(\nabla \varphi_{e}\right)=\sigma_{0}+\frac{\sigma_{1}}{1+d \exp \left(-\frac{\left|\nabla \varphi_{e}\right|-a}{b}\right)}
$$


where the parameters $\sigma_{0}, \sigma_{1}, \mathrm{a} ; \mathrm{b}$ and d depend on the type of biological tissue. When electroporation occurs, the tissue conductivity increases in the area between the electrodes (see Fig. 3 top).

Considering a nonlinear conductivity $\sigma_{\mathrm{e}}\left(\nabla \varphi_{\mathrm{e}}\right)$ in (1), it should not be possible to rigorously formulate the zero and first order of the asymptotic solution as proposed in section II. However, since iterative techniques are necessary used to solve a nonlinear problem, the asymptotic development can be applied at each iteration. In this paper, Picard's algorithm [8] is used to compute the solution $\varphi=\varphi_{\mathrm{e}}+\varphi_{\mathrm{i}}$ in the nonlinear problem. From the solution $\varphi^{\mathrm{k}-1}$ computed at the iteration $\mathrm{k}-1$, the problems (4a)-(4b) are solved with $\sigma_{e}\left(\nabla \varphi^{k-1}\right)$ to calculate the intermediate solution $\varphi_{\mathrm{e}+\mathrm{i}}{ }^{\mathrm{k}}=\varphi_{\mathrm{e}}^{\mathrm{k}}+\varphi_{\mathrm{i}}^{\mathrm{k}}$. Then, a relaxation factor $\mathrm{K}^{\mathrm{k}}$ is applied to optimize the contribution of the intermediate solution $\varphi_{\mathrm{e}+\mathrm{i}}{ }^{\mathrm{k}}$ to the solution $\varphi^{\mathrm{k}}=\varphi^{\mathrm{k}-1}+\mathrm{K}^{\mathrm{k}}\left(\varphi_{\mathrm{e}+\mathrm{i}}^{\mathrm{k}}-\varphi^{\mathrm{k}-1}\right)$ computed at the iteration $\mathrm{k}$; note that $\mathrm{K}^{\mathrm{k}}$ is corrected at each iteration according to how the solution is approached in order to accelerate and stabilize the convergence [8].

Picard's algorithm has been performed to solve the nonlinear problem for both the direct solution and the asymptotic solution at the zero order, in the case where the conductivity $\sigma_{i}=10^{3} \sigma_{0}$ is set for the passive electrodes. In order to make a mindful comparison, the 2D model has been chosen with the fine mesh. The algorithm stops when the relative error between solutions $\varphi^{\mathrm{k}-1}$ and $\varphi^{\mathrm{k}}$ reaches $10^{-8}: 23$ iterations are required for the asymptotic approach and 33 iterations for the direct solution. The relative error on $\mathrm{R}$ is $1.210^{-3}$ with the asymptotic development at the zero order, which is 22 times larger than the error obtained in the linear case. Fig. 5 shows that the relative error on the conductivity between the direct solution and the asymptotic approach reaches $7.10^{-3}$ in the electroporated region.

\section{IV.CONCLUSION}

In this paper, an asymptotic approach has been proposed to solve the problem of floating potentials that present a high conductivity compared to the ambient medium. The convergence study shows that this approach enables to improve the accuracy of the solution where perfect conductors are assumed. Compared to the direct solution, the advantage of the asymptotic approach is that less numerical resources are required since the floating conductors are meshed in a distinct problem. Another advantage of the asymptotic approach is that it avoids numerical artifacts that appear with the direct solution when the contrast of conductivity is too large. Nonlinear problems can also be solved numerically using the asymptotic method: a realistic example shows that the asymptotic solution at the zero order introduces a larger error compared to the linear case.

\section{ACKNOWLEDGEMENT}

This research has been carried out thanks to the support of the research projects DYNAMO and NUMEP granted by Plan Cancer. This work lies in the scope of the Inria Associate team NUM4SEP.

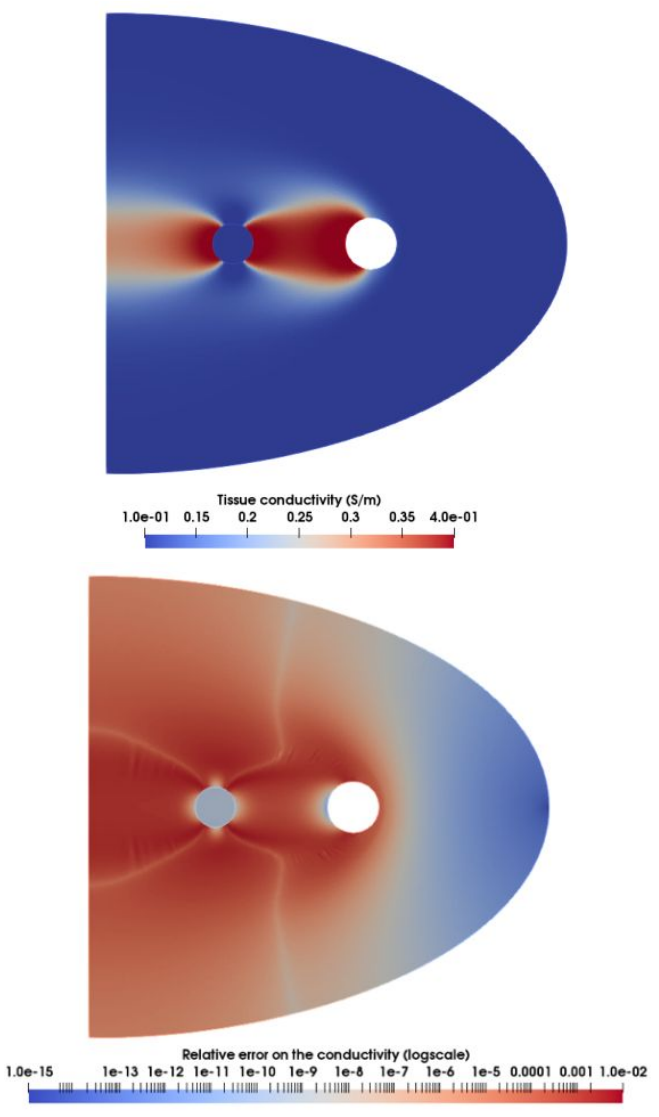

Fig.5: (top) Distribution of the conductivity computed using the asymptotic approach (bottom) Relative error on the conductivity between the asymptotic approach and the direct solution. $\mathrm{V}=138$ Volts in (1) and the tissue parameters are $\sigma_{0}=0.1 \mathrm{~S} / \mathrm{m}, \sigma_{1}=0.3 \mathrm{~S} / \mathrm{m}, \mathrm{a}=\left(\mathrm{E}_{0}+\mathrm{E}_{1}\right) / 2 ; \mathrm{b}=\left(\mathrm{E}_{1}-\mathrm{E}_{0}\right) / \mathrm{c}, \mathrm{c}=8$ and $\mathrm{d}=10$ with $\mathrm{E}_{0}=460 \mathrm{~V} / \mathrm{cm}$ and $\mathrm{E}_{1}=700 \mathrm{~V} / \mathrm{cm}$.

\section{REFERENCES}

[1] A. Konrad and M. Graovac, The Finite Element Modeling of Conductors and Floating Potentials, IEEE Transactions on Magnetics, Vol. 32, No. 5, 1996.

[2] R. V. Sabariego, M. V. Ferreira da Luz, J. P. Nzuru Nsekere, P. Kuo-Peng, J. L. Lilien and P. Dular, Perturbation Finite Element Method for the Analysis of Earthing Systems with Vertical Rods, Proceedings of MOMAG, 2008.

[3] T. Ivorra, B. Al-Sakere, B. Rubinsky and L. M Mir, In vivo electrical conductivity measurements during and after tumor electroporation: conductivity changes reflect the treatment outcome, Physics in Biology and Medecine, Vol. 54, 2009.

[4] D. Amann, A. Blaszczyk, G. Of and O. Steinbach, Simulation of floating potentials in industrial applications by boundary element methods, Journal of Mathematics in Industry, 2014.

[5] W. N. Wu, S. L. Ho, S. Niu and J. Zhu, Comparison Study of Finite Element Methods to Deal With Floating Conductors in Electric Field, IEEE Transaction on Magnetics,, Vol. 48, No. 2, 2012

[6] W. Yu, M. Zhang, and Z. Wang, Efficient 3-D Extraction of Interconnect Capacitance Considering Floating Metal Fills With Boundary Element Method, IEEE Transactions on Computer Aided Design of Integrated Circuits and Systems, Vol. 25, No. 1, 2006

[7] G. Aiello, S. Alfonzetti, S. A. Rizzo, and N. Salerno, FEM-DBCI Solution of Open-Boundary Electrostatic Problems in the Presence of Floating Potential Conductors, IEEE Transactions on Magnetics, Vol. 52, No. 3, 2016.

[8] M. Breton, F. Buret, L. Krähenbühl, M. Leguèbe, L. M. Mir, et al., Nonlinear steady-state electrical current modeling for the electropermeabilization of biological tissue, 16th Biennial IEEE Conference on Electromagnetic Field Computation, 2014. 